\title{
Article \\ Post-Synthesis Modification of Photoluminescent and Electrochemiluminescent Au Nanoclusters with Dopamine
}

\author{
Jae Hyun Kim ${ }^{1}$ and Joohoon Kim ${ }^{1,2, *(D)}$ \\ 1 Department of Chemistry, Research Institute for Basic Science, Kyung Hee University, Seoul 02447, Korea; \\ blangka12@khu.ac.kr \\ 2 KHU-KIST Department of Converging Science and Technology, Kyung Hee University, Seoul 02447, Korea \\ * Correspondence: jkim94@khu.ac.kr; Tel.: +82-2-961-9384
}

Citation: Kim, J.H.; Kim, J. Post-Synthesis Modification of Photoluminescent and Electrochemiluminescent $\mathrm{Au}$ Nanoclusters with Dopamine. Nanomaterials 2021, 11, 46. https://dx.doi.org/10.3390/ nano11010046

Received: 4 December 2020 Accepted: 24 December 2020 Published: 27 December 2020

Publisher's Note: MDPI stays neutral with regard to jurisdictional claims in published maps and institutional affiliations.

Copyright: () 2020 by the authors. Licensee MDPI, Basel, Switzerland. This article is an open access article distributed under the terms and conditions of the Creative Commons Attribution (CC BY) license (https: / / creativecommons.org/ licenses/by/4.0/).

\begin{abstract}
Here, we report a post-synthesis functionalization of the shell of Au nanoclusters (NCs) synthesized using glutathione as a thiolate ligand. The as-synthesized Au NCs are subjected to the post-synthesis functionalization via amidic coupling of dopamine on the cluster shell to tailor photoluminescence (PL) and electrochemiluminescence (ECL) features of the Au NCs. Because the NCs' PL at ca. $610 \mathrm{~nm}$ is primarily ascribed to the $\mathrm{Au}(\mathrm{I})$-thiolate (SG) motifs on the cluster shell of the NCs, the post-synthesis functionalization of the cluster shell enhanced the PL intensity of the $\mathrm{Au}$ NCs via rigidification of the cluster shell. In contrast to the PL enhancement, the post-synthesis modification of the cluster shell does not enhance the near-infrared (NIR) ECL of the NCs because the NIR ECL at $c a .800 \mathrm{~nm}$ is ascribed to the Au(0)-SG motifs in the metallic core of the NCs.
\end{abstract}

Keywords: post-synthesis modification; Au nanoclusters; dopamine; photoluminescence; electrochemiluminescence

\section{Introduction}

$\mathrm{Au}$ nanoclusters ( $\mathrm{Au} \mathrm{NCs}$ ) comprising a small number of atoms, typically with a particle size of $\sim 1 \mathrm{~nm}$, have attracted intense research in both fundamental and applied disciplines as a promising class of functional nanomaterials [1-3]. Recently, Au NCs have thus risen to the forefront of sensing, photovoltaic, and diagnostic research [4-8]. This is because the ultra-small Au NCs exhibit unique molecular-like behaviors, such as photoluminescence (PL), molecular chirality, quantized double-layer charging, and electrochemiluminescence (ECL), distinctively different from their larger nanoparticle and bulk counterparts [9-12]. These distinctive properties were found to be highly dependent on the size, oxidation states, composition, and ligands of the Au NCs [13-16].

A variety of ligands, such as alkanethiols, dendrimers, peptides, and proteins, have been used to synthesize well-defined Au NCs. Among the various ligands studied so far, a hydrophilic tripeptide glutathione (GSH) has been particularly useful for synthesizing $\mathrm{Au}$ NCs in aqueous medium with rational tuning of their properties $[17,18]$. For example, Negishi et al. synthesized a series of GSH-protected Au NCs ranging from $\mathrm{Au}_{10}(\mathrm{SG})_{10}$ to $\mathrm{Au}_{39}(\mathrm{SG})_{24}$ [19]. The GSH-protected Au NCs exhibited PL in the visible to near-infrared (NIR) region [20-22], and PL quantum yield of $8 \%$ was observed for $\mathrm{Au}_{22}(\mathrm{SG})_{18}$ in water [20]. In addition, GSH-protected Au NCs were reported to exhibit intense ECL with an ECL quantum yield as high as $0.42 \%$ [23]. To tailor the photophysical/electrochemical properties of Au NCs in rational ways, various surface engineering approaches have been suggested, such as rigidification of the cluster shell, exchange of surface ligands on the cluster shell, surface functionalization of the cluster shell with light-harvesting ligands, and self-assembly of Au NCs through their ligand shells [24,25]. For example, rigidification of $\mathrm{Au}(\mathrm{I})$-thiolate shell of $\mathrm{Au}$ NCs was proposed as a strategy to amplify the PL efficiencies of Au NCs [21,26], and ligand exchange was demonstrated as a methodology to enhance PL quantum yield of Au NCs stabilized by phenylethanethiolate ligands upon 
the ligand exchange with 1,4-dithiol durene [27]. Because brightness of Au NCs depends on the absorption efficiency of excitation light as well as the PL quantum yield of the NCs, light-harvesting ligands (i.e., chromophores) were introduced on the cluster shell of $\mathrm{Au}$ NCs to efficiently adsorb excitation light and to transfer the excitation energy from the surface-bound chromophores to the emitting Au NCs [28]. More recently, cationic polymermediated self-assembly of Au NCs was demonstrated for preparing a highly luminescent assembly of Au NCs depending on NCs' surface ligands and cationic polymers [29].

Here, we report a post-synthesis functionalization of the shell of Au NCs with dopamine to investigate how the surface functionalization of Au NCs affects both PL and ECL of the Au NCs. The Au NCs were synthesized using GSH acting as a thiolate ligand, similar to previous reports [18,30]. In agreement with our recent studies, the as-synthesized $\mathrm{Au}$ NCs exhibited a strong NIR ECL peak with a shoulder peak in the visible region, while the nanoclusters emitted a strong PL peak primarily in the visible region $[30,31]$. We ascribed the NIR and visible emission peaks of the nanoclusters to the $\mathrm{Au}(0)$-SG motif in their metallic core and the $\mathrm{Au}(\mathrm{I})$-SG motif on their shell surface, respectively $[30,31]$. The as-synthesized $\mathrm{Au} \mathrm{NCs}$ were subjected to the post-synthesis functionalization for surface coupling of dopamine on the cluster shell. Spectroscopic measurements including ultraviolet-visible (UV-vis) absorption, Fourier-transform infrared (FT-IR), and nuclear magnetic resonance (NMR) confirmed the successful coupling of dopamine. Interestingly, the surface functionalization enhanced the PL intensity of the $\mathrm{Au}$ NCs but weakened the NIR ECL of the clusters. This finding is important because it demonstrates the significance of surface-engineered Au NCs to tailor the clusters' PL and ECL as a post-synthesis functionalization of the cluster shell.

\section{Materials and Methods}

\subsection{Materials}

All chemicals were used as received without further purification. The following chemicals were received from Sigma Aldrich, Inc. (St. Louis, MO, USA): gold(III) chloride trihydrate $\left(\mathrm{HAuCl}_{4} \cdot 3 \mathrm{H}_{2} \mathrm{O}, \geq 99.9 \%\right.$, trace metals basis), $\mathrm{L}$-glutathione reduced $(\mathrm{GSH}$, $\geq 98.0 \%$ ), 9-fluorenylmethoxycarbonyl chloride (Fmoc chloride, 97\%), tetrahydrofuran (THF, $\geq 99.9 \%$, anhydrous), dopamine hydrochloride, potassium nitrate $\left(\mathrm{KNO}_{3}, 99.999 \%\right)$, phosphate-buffered saline (PBS) buffer, 1-[3-(dimethylamino)propyl]-3-ethylcarbodiimide methiodide (EDC), $N$-hydroxy-succinimide (NHS, 98\%), triethylamine (TEA, $\geq 99 \%$ ), gold standard for ICP $(1000 \mathrm{mg} / \mathrm{L} \mathrm{Au}$ in $\mathrm{HCl})$, and deuterium oxide $\left(\mathrm{D}_{2} \mathrm{O}, 99.9\right.$ atom \% D). Sodium bicarbonate $\left(\mathrm{NaHCO}_{3}, \geq 99.0 \%\right.$ ) was obtained from Daejung Inc. (Gyeonggi, Korea). Hydrochloric acid $(\mathrm{HCl}, 35.0 \sim 37.0 \%)$ and nitric acid $\left(\mathrm{HNO}_{3}, 60.0 \%\right)$ were purchased from Samchun Chemical Co., Ltd. (Seoul, Korea). Amicon ${ }^{\circledR}$ Ultra-4 centrifugal ultrafiltration filters (3 kDa MWCO) were purchased from Merck Millipore Corp. (Danvers, MA, USA). Deionized water (DI water, $18 \mathrm{M} \Omega \cdot \mathrm{cm}$ ) was used in the preparation of aqueous solutions (Aquapuri541, YOUNG IN Chromass, Gyeonggi, Korea).

\subsection{Synthesis and Post-Synthesis Modification of NCs}

$\mathrm{Au}$ NCs were synthesized using GSH as both a reductant and stabilizer by following a previously reported procedure $[18,30]$. In brief, fresh aqueous solutions of $100 \mathrm{mM} \mathrm{GSH}$ $(0.6 \mathrm{~mL})$ and $20 \mathrm{mM} \mathrm{HAuCl}{ }_{4} \cdot 3 \mathrm{H}_{2} \mathrm{O}(2.0 \mathrm{~mL})$ were added to $17.4 \mathrm{~mL}$ of DI water under stirring. The aqueous mixture was kept under vigorous stirring at room temperature for $1 \mathrm{~min}$ and subsequently heated to $70^{\circ} \mathrm{C}$ for $24 \mathrm{~h}$. After following this synthesis procedure, the as-synthesized $\mathrm{Au}$ NCs were subjected to a post-synthesis modification as follows: $180 \mathrm{mmol}$ of Fmoc chloride was dissolved in $1.0 \mathrm{~mL}$ of THF containing $1 \mathrm{mg}$ of $\mathrm{NaHCO}_{3}$. This solution was added to $1.0 \mathrm{~mL}$ of the as-synthesized Au NCs $(10 \mathrm{mg} / \mathrm{mL})$ in DI water. After the solution was stirred vigorously at room temperature for $3 \mathrm{~h}, 8.0 \mathrm{~mL}$ of THF was added to the mixture, followed by centrifugation at $3600 \mathrm{rpm}$ for $15 \mathrm{~min}$ to precipitate Fmoc-protected Au NCs that had formed. The collected Fmoc-protected Au NCs were washed twice with THF and then dried overnight, after which $10 \mathrm{mg}$ of purified Fmoc- 
protected $\mathrm{Au}$ NCs were re-dissolved in $2.0 \mathrm{~mL}$ of PBS buffer (pH 7.4). Then, $60 \mathrm{mmol}$ each of EDC and NHS were added to $2.0 \mathrm{~mL}$ of Fmoc-protected Au NCs $(5.0 \mathrm{mg} / \mathrm{mL})$ and kept under stirring at room temperature for $20 \mathrm{~min}$; then, $120 \mathrm{mmol}$ of dopamine hydrochloride dissolved in DI water was added. After the mixture was stirred for $16 \mathrm{~h}$, the cluster product was purified with centrifugal ultrafiltration filters ( $3 \mathrm{kDa} \mathrm{MWCO}$ ), resulting in dopamine-conjugated Au NCs.

\subsection{Characterization}

UV-vis absorption and PL spectra were recorded using an Agilent $8453 \mathrm{UV}$-vis spectrometer (Agilent Tech., Wilmington, DE, USA) and a FS-2 fluorescence spectrometer (Scinco Co., Seoul, Korea), respectively. Absolute PL quantum yields were determined using a Quantaurus-QY C11347 spectrometer (Hamamatsu Photonics K.K., Shizuoka, Japan). Inductively coupled plasma-atomic emission spectroscopy (ICP-AES) was carried out to determine the GSH-to-Au ratio of clusters using an iCAP ${ }^{\mathrm{TM}} 7200$ spectrometer (Thermo Scientific, Waltham, MA, USA). Transmission electron microscopy (TEM) was performed using a Tecnai G ${ }^{2}$ F30 S-Twin instrument (FEI Co., Hillsboro, OR, USA) operating at $200 \mathrm{kV}$. TEM samples were prepared by dropping aqueous cluster solutions onto 200 mesh carbon-coated Ni grids (Ted Pella Inc., Redding, CA, USA) followed by drying in air. NMR spectroscopy was carried out with an Avance III HD 400 spectrometer (Bruker Corp., Billerica, MA, USA). NMR samples were prepared by dissolving clusters in $\mathrm{D}_{2} \mathrm{O}$. FT-IR spectroscopy was conducted on an Alpha II spectrometer (Bruker Corp., Billerica, MA, USA). Dynamic light scattering (DLS) measurements were performed with a Zetasizer Nano ZS90 molecular analyzer (Malvern Panalytical, Malvern, UK). ECL spectra were recorded using a three-electrode electrochemical cell connected to the slit of an Acton Standard SP2150 monochromator (Princeton Instruments, Trenton, NJ, USA) equipped with a PIXIS 100B charge-coupled device (CCD) camera (Princeton Instruments, Trenton, NJ, USA). For all electrochemical measurements, we used a $\mathrm{CHI} 440 \mathrm{~A}$ potentiostat $(\mathrm{CH}$ Instruments Inc., Bee Cave, TX, USA) with the three-electrode cell, and we used a glassy carbon electrode (dia.: $3 \mathrm{~mm}$ ) as the working electrode. We used an $\mathrm{Ag} / \mathrm{AgCl}(3 \mathrm{M} \mathrm{NaCl})$ and a coiled $\mathrm{Pt}$ wire as the reference electrode and the auxiliary electrode, respectively.

\section{Results and Discussion}

As shown in Figure 1a, we protected the amino groups of GSH on the shell of the as-synthesized Au NCs with Fmoc chloride. The resulting Fmoc-protected Au NCs were then functionalized via covalent coupling of dopamine to carboxyl groups of GSH on the cluster shell (see Materials and Methods Section for details). Figure 1b shows UVvis absorption spectra of the as-synthesized Au NCs and the dopamine-conjugated $\mathrm{Au}$ NCs. The dopamine-conjugated Au NCs were pale yellow in aqueous solution, while the as-synthesized Au NCs were bright yellow (inset in Figure 1b). The as-synthesized Au NCs ((i) in Figure 1b) exhibited a broad UV-vis absorption peak at $c a .400 \mathrm{~nm}$ but did not show the surface plasmon absorption band typically observed for Au nanoparticles larger than $2 \mathrm{~nm}$ [32]. These UV-vis absorption characteristics agreed with those observed for small GSH-protected Au NCs that emit orange PL with a maximum peak at ca. $610 \mathrm{~nm}$ via aggregation-induced emission (AIE) of Au(I)-SG motif in the compact shell of the NCs [18]. The content of GSH in the as-synthesized Au NCs was quantified by determining the GSH-to-Au ratio in the clusters. ICP-AES analysis revealed that GSH contributed 54\% of the as-synthesized Au NCs by weight. This result could be translated to a GSH-to-Au ratio of $0.76: 1$, which was consistent with the value (i.e., $0.84: 1$ ) previously reported by thermal gravimetric analysis (TGA) of the GSH-protected Au NCs [18]. For the dopamineconjugated Au NCs ((ii) in Figure 1b), we observed a new UV-vis absorption shoulder peak at $c a .270 \mathrm{~nm}$, which is similar to that of dopamine (Supplementary Materials, Figure S1), thus indicating successful surface coupling of dopamine on the shell surface of NCs. 


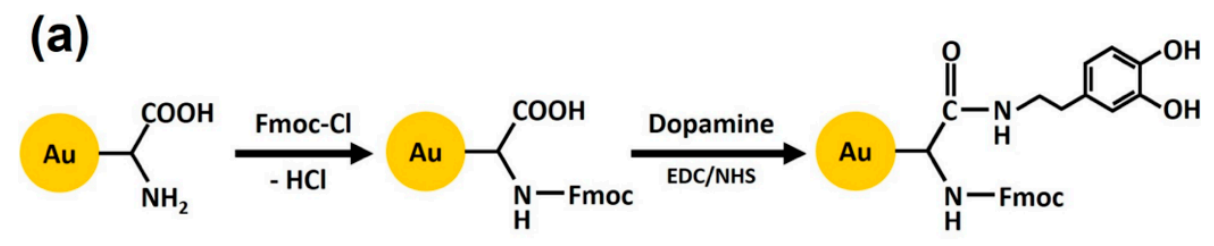

(b)

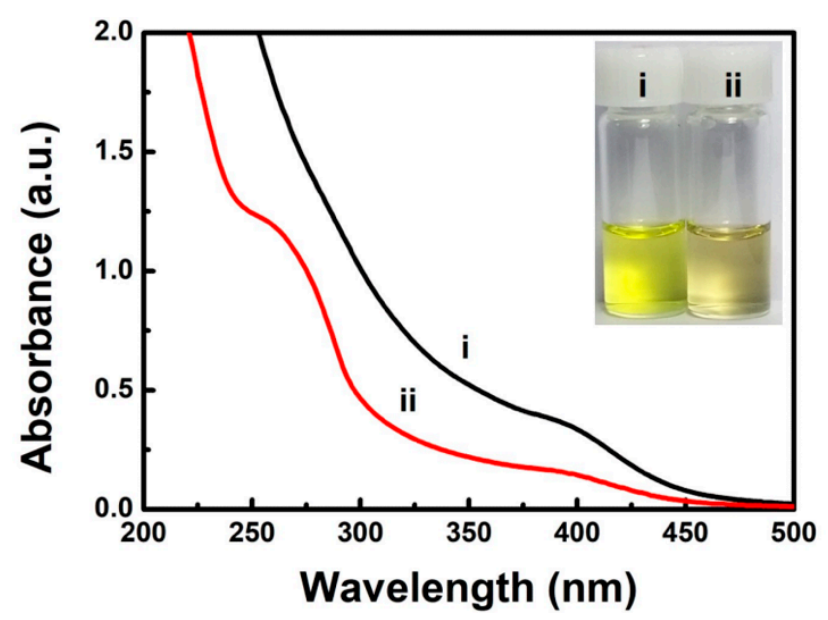

Figure 1. (a) Schematic illustration of post-synthesis functionalization of as-synthesized Au NCs with dopamine. (b) UV-vis absorption spectra of (i) as-synthesized Au NCs and (ii) dopamine-conjugated $\mathrm{Au}$ NCs. The inset shows a digital photo of as-synthesized Au NCs and dopamine-conjugated $\mathrm{Au}$ NCs in water under visible light. Concentration of Au NCs: $1.5 \mathrm{mg} / \mathrm{mL}$.

In addition to the UV-vis absorption measurements, we performed FT-IR and NMR studies to confirm the covalent coupling of dopamine to carboxyl groups of GSH on the cluster shell. Figure 2 shows FT-IR spectra of free GSH and the as-synthesized Au NCs. Unlike the free GSH counterpart ((i) in Figure 2), the as-synthesized Au NCs did not show a band at $\mathrm{ca} .2522 \mathrm{~cm}^{-1}$, corresponding to S-H stretching vibration mode, in their FT-IR spectrum ((ii) in Figure 2) [33]. The disappearance of the FT-IR band at $2522 \mathrm{~cm}^{-1}$ in the as-synthesized Au NCs indicates the adsorption of GSH in the form of the thiolate (GS) to the Au surface via the Au-S bond formation, as reported previously [19]. Other characteristic FT-IR bands of the as-synthesized Au NCs appeared wider but otherwise roughly the same as those in the FT-IR spectrum of the free GSH, which further suggests GSH ligation to the Au surface. The main characteristic bands are the absorption bands of $\mathrm{C}=\mathrm{O}$ stretching mode at $c a .1714 \mathrm{~cm}^{-1}$, amide I mode (mainly $\mathrm{C}=\mathrm{O}$ stretching) at $c a .1642$ $\mathrm{cm}^{-1}$, amide II mode (mainly N-H bending and C-N stretching) at $c a .1529 \mathrm{~cm}^{-1}$, and C-O stretching mode at $c a .1397 \mathrm{~cm}^{-1}$. All of these bands indicate the presence of GSH adsorbed on the Au surface. The as-synthesized Au NCs were subjected to post-synthesis modification for the covalent coupling of dopamine via amidic coupling to carboxyl groups on the cluster shell. Compared with the FT-IR spectrum of the as-synthesized Au NCs ((ii) in Figure 3), the prominent band at $c a .1714 \mathrm{~cm}^{-1}$, attributed to the $\mathrm{C}=\mathrm{O}$ stretching band of carboxyl moieties of GSH, vanished upon the covalent coupling of dopamine to the surface carboxyl groups ((iii) in Figure 3), which indicates the covalent conjugation of dopamine on the cluster shell after the post-synthesis modification. We also verified the presence of conjugated dopamine in the FT-IR spectrum of the dopamine-conjugated Au NCs with the appearance of FT-IR bands at $c a .1603 \mathrm{~cm}^{-1}$ corresponding to C-C bonds in the aromatic ring of dopamine ((i) and (iii) in Figure 3). Furthermore, ${ }^{1} \mathrm{H}$ NMR spectrum of the as-synthesized $\mathrm{Au}$ NCs shows major peaks corresponding to methylene groups of GSH (Supplementary Materials, Figure S2a), indicating the passivation of the clusters with GSH, as reported previously [26]. More specifically, by comparison with previous NMR studies of free GSH and GSH-stabilized Au NCs [34,35], the merged broad peaks around $\sim 3.9 \mathrm{ppm}$ 
were assigned to the $\mathrm{CH}_{2}$ at $\mathrm{C}-9$ and $\mathrm{CH}$ at $\mathrm{C}-1$, as shown in Figure S2a (Supplementary Materials). The broad multiplets at 2.1 and $2.5 \mathrm{ppm}$ were also assigned to the $\mathrm{CH}_{2}$ at $\mathrm{C}-2$ and C-3, respectively. Note that the minor peaks at $2.8-3.2 \mathrm{ppm}$ and $2.3 \mathrm{ppm}$ were from residual glutathione disulfide (GS-SG), as reported previously [36]. In addition, the ${ }^{1} \mathrm{H}$ NMR spectrum of the dopamine-conjugated Au NCs shows peaks related to the aromatic and aliphatic protons of dopamine (Supplementary Materials, Figure S2b), verifying the surface coupling of dopamine on the cluster shell after the post-synthesis functionalization. The ${ }^{1} \mathrm{H}$ NMR spectrum also displays no detectable peaks related to dihydroxyindole (DHI), known as a key precursor involved in the oxidative polymerization of dopamine to polydopamine, indicating the absence of polydopamine in the dopamine-conjugated $\mathrm{Au}$ NCs $[37,38]$. The integration of peak intensities in the ${ }^{1} \mathrm{H}$ NMR spectrum of the dopamineconjugated $\mathrm{Au}$ NCs provides the estimated molar ratio of coupled dopamine to GSH (i.e., $\sim 0.8$ ) on the cluster shell.



Figure 2. FT-IR spectra of (i) free GSH and (ii) as-synthesized Au NCs in the $1000-2800 \mathrm{~cm}^{-1}$ region.

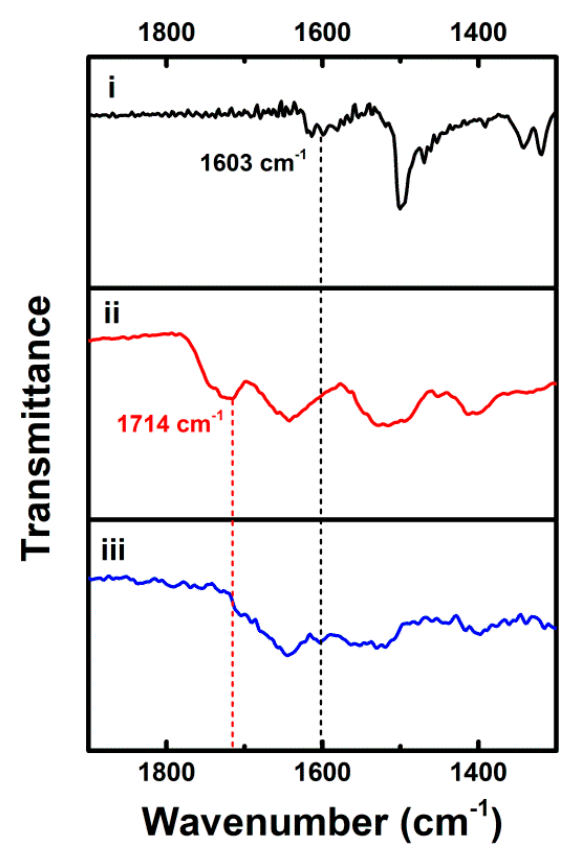

Figure 3. FT-IR spectra of (i) free dopamine, (ii) as-synthesized Au NCs, and (iii) dopamineconjugated Au NCs in the $1300-1900 \mathrm{~cm}^{-1}$ region. 
Figure 4a shows a TEM image of the as-synthesized Au NCs, indicating that the NCs were ultra-small and fairly uniform in size, as reported previously for Au NCs synthesized using GSH [18,39]. The size distribution histogram of the NCs revealed a narrow size distribution of the Au metal core with an average size of $1.2 \pm 0.2 \mathrm{~nm}$ (inset in Figure 4a). The measured small size of the as-synthesized Au NCs (i.e., less than $2 \mathrm{~nm}$ ) was also supported by the absence of the Au surface plasmon absorption band in the UV-vis absorption spectrum of the NCs, as discussed earlier ((i) in Figure 1b). Interestingly, as shown in Figure $4 \mathrm{~b}$, the measured size of the dopamine-conjugated Au NCs (i.e., $1.2 \pm 0.1 \mathrm{~nm}$ ) did not differ significantly from that of the as-synthesized Au NCs, which indicates no significant aggregation of the NCs even after the covalent conjugation of dopamine on the cluster shell. In contrast, DLS measurements of both the as-synthesized and the dopamineconjugated $\mathrm{Au} \mathrm{NCs}$ revealed a significant change in the clusters' hydrodynamic diameters from $1.8 \pm 1.2 \mathrm{~nm}$ for the as-synthesized Au NCs to $627.1 \pm 147.9 \mathrm{~nm}$ for the dopamineconjugated Au NCs. Because little aggregation of Au metal core occurred in the clusters (Figure 4), this substantial change in their hydrodynamic diameters was primarily attributed to the dopamine conjugation to GSH on the cluster shell [40]. These results indicate that the post-synthesis functionalization of the as-synthesized Au NCs led to successful coupling of dopamine on the cluster shell, while keeping the NCs from aggregating.
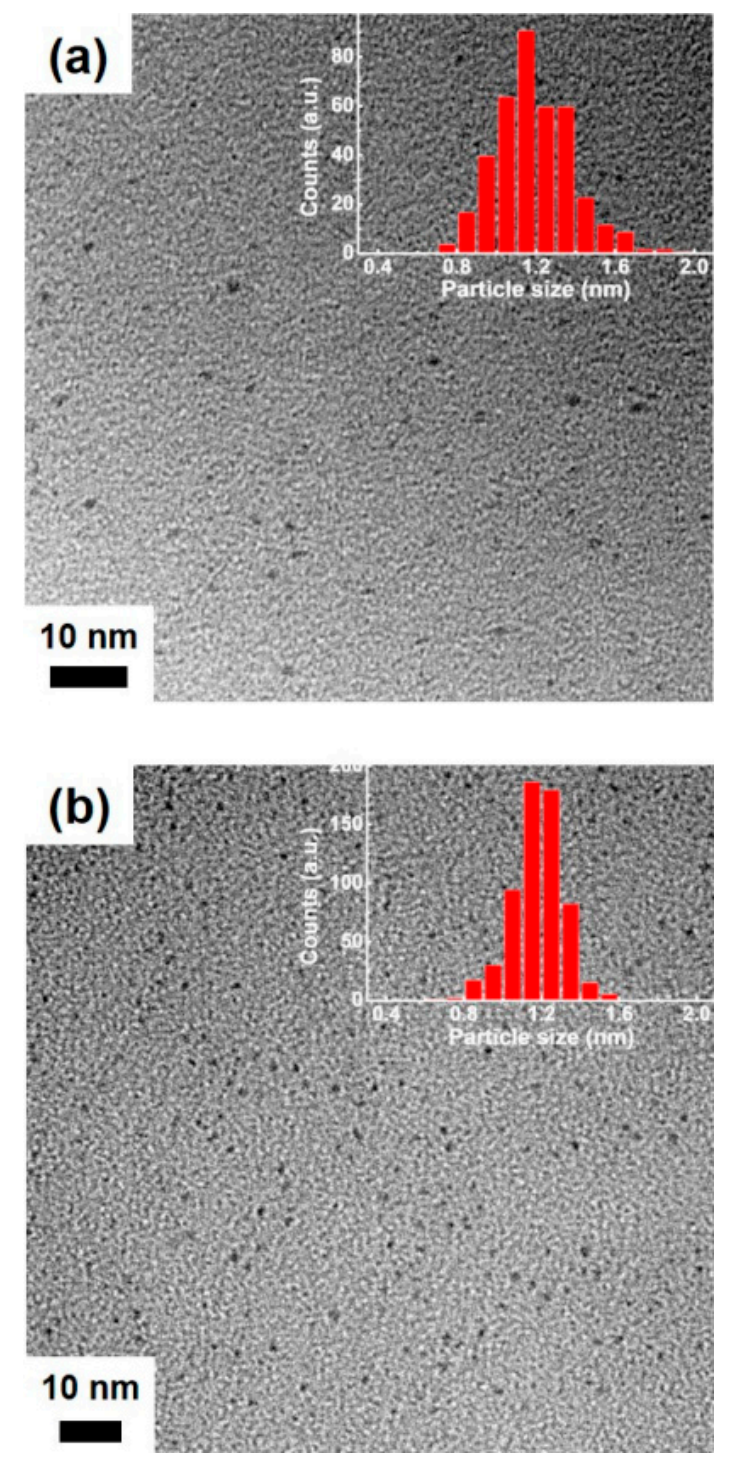

Figure 4. TEM images of (a) as-synthesized Au NCs and (b) dopamine-conjugated Au NCs. The insets of (a) and (b) show size distribution histograms of the NCs. 
We measured PL and ECL to investigate the influence of the post-synthesis functionalization of the Au NC shells with dopamine on the clusters' photophysical and electrochemical properties. Figure 5a shows PL spectra of the as-synthesized Au NCs and the dopamine-conjugated Au NCs. The as-synthesized Au NCs exhibited orange PL with a maximum peak at $c a .610 \mathrm{~nm}$, with a weak shoulder peak at $c a .790 \mathrm{~nm}$ upon excitation at $\lambda_{\text {ex }}=365 \mathrm{~nm}$ ((i) in Figure 5a). The orange PL of the as-synthesized Au NCs at $610 \mathrm{~nm}$ was in agreement with the PL emission observed for the Au NCs synthesized using GSH in previous reports, wherein the PL was attributed to AIE of the Au(I)-SG motifs on their shell surface $[18,30,31]$. The PL emission could be ascribed to ligand-to-metal charge transfer (LMCT) or ligand-to-metal-metal charge transfer (LMMCT) from the sulfur atom to the Au atoms in the dense $\mathrm{Au}(\mathrm{I})-\mathrm{SG}$ shell on the cluster surface, and subsequent radiative relaxation $[18,41]$. After the post-synthesis functionalization of the as-synthesized Au NCs with dopamine, we observed the increase in PL intensity of the clusters, especially at $610 \mathrm{~nm}$ ((ii) in Figure 5a). Because the orange PL at $610 \mathrm{~nm}$ was ascribed to the Au(I)-SG motifs on the NC surface, the observed increase in PL intensity of the dopamine-conjugated Au NCs suggested dopamine conjugation on the NC surface via the post-synthesis functionalization of the NCs. The PL enhancement of the dopamine-conjugated Au NCs primarily at $610 \mathrm{~nm}$ also suggested that the electron-donating capability of the ligands on the dopamineconjugated Au NCs did not play a major role in the PL enhancement of the clusters despite the possible ligand to metal nanoparticle core charge transfer (LMNCT) previously reported [16]. This is most likely because the orange PL at $610 \mathrm{~nm}$ was attributed to AIE of $\mathrm{Au}(\mathrm{I})-\mathrm{SG}$ motif in the shell of the NCs, as discussed earlier. By integrating PL intensities of the clusters over the wavelength, we observed an increase of 1.5 times in the PL emission of the dopamine-conjugated Au NCs over that of the as-synthesized clusters. In addition, the PL quantum yields of the as-synthesized Au NCs and the dopamine-conjugated $\mathrm{Au}$ NCs were found to be $1.9 \%$ and $2.6 \%$, respectively. In recent studies, investigators suggested rigidification of the $\mathrm{Au}(\mathrm{I})$-thiolate shell of $\mathrm{Au}$ NCs as an effective strategy to enhance the PL of Au NCs [21,26,42]. For example, Lee and coworkers demonstrated PL enhancement of GSH-protected $\mathrm{Au}$ NCs (i.e., $\mathrm{Au}_{22}(\mathrm{SG})_{18}$ ) by rigidifying the shells of NCs with lipophilic bulky tetraoctylammonium cations [21]. More recently, a similar PL enhancement was reported by rigidifying the shell of $\mathrm{Au}_{22}(\mathrm{SG})_{18}$ via covalent modification of the SG ligand shells on the clusters with aromatic pyrene chromophores [26]. Therefore, we attributed the observed PL enhancement for the dopamine-conjugated Au NCs to shell rigidification that arose from the $\pi-\pi$ interactions between aromatic catechol moieties of dopamine conjugated on the cluster shell. It is noteworthy that the PL enhancement for the dopamine-conjugated Au NCs might be attributable to AIE caused by the dopamine conjugation on the NCs [43-45]. However, we ruled out the possibility of PL enhancement by AIE because TEM measurements of the dopamine-conjugated Au NCs showed little aggregation of the NCs, as discussed earlier. In addition to the PL emission spectra, we measured PL excitation spectra of the as-synthesized Au NCs and the dopamine-conjugated Au NCs (Supplementary Materials, Figure S3). Figure S3 shows the PL excitation spectra displaying a maximum around $c a .400 \mathrm{~nm}$, which coincided with the broad absorption shoulder observed in the UV-vis absorption spectra of the NCs (Figure 1b). Meanwhile, Figure 5b shows ECL spectra of the as-synthesized Au NCs and the dopamine-conjugated $\mathrm{Au}$ NCs. Their ECL spectra were obtained at a potential of $1.3 \mathrm{~V}$ in the presence of TEA as an anodic co-reactant for oxidative-reduction ECL reactions. In agreement with previous studies [30,31], the ECL spectra show a strong NIR ECL peak at ca. $800 \mathrm{~nm}$ with a shoulder peak at $c a .620 \mathrm{~nm}$. Because the shoulder peak wavelength matched the clusters' orange PL, we attributed the shoulder ECL emission to the Au(I)-SG motifs on the NC surface, as reported in the previous findings [30,31]. Similarly, the NIR ECL emission was ascribed to the $\mathrm{Au}(0)$-SG motifs, responsible for the PL of the clusters at $790 \mathrm{~nm}$, in the metallic core of the Au NCs [30,31]. Feasible electrochemical oxidation of the Au(0)-SG motifs, in contrast with that of the $\mathrm{Au}(\mathrm{I})$-SG motifs, was responsible for the clusters' strong NIR ECL emission. In contrast to the PL enhancement, after functionalizing the cluster shell 
of the as-synthesized Au NCs with dopamine, we observed decreased ECL intensity of the clusters. Because the $\mathrm{Au}(0)-\mathrm{SG}$ motifs in the cluster core were responsible for their NIR ECL, the diminished ECL emission of the dopamine-conjugated Au NCs suggested that the possible LMNCT previously reported did not play a significant role in the ECL enhancement of the clusters, as we discussed for their PL enhancement earlier [16]. Instead, the dopamine-conjugated Au NCs showed diminished ECL emission ((ii) in Figure 5b), presumably attributable to deteriorated electrical conductivity, which limited the electrochemical oxidation required for anodic ECL generation of the NCs with the organic dopamine conjugated on the cluster surface.
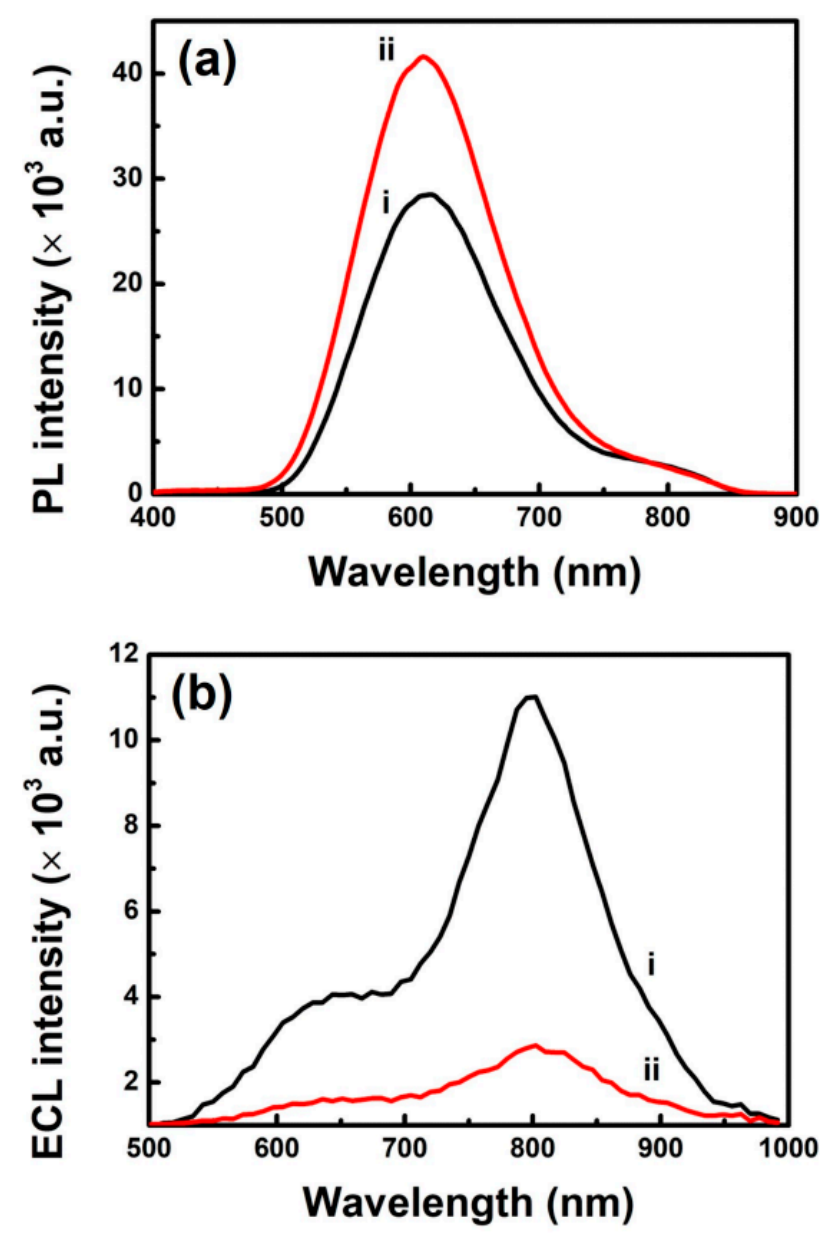

Figure 5. (a) PL emission spectra of (i) as-synthesized Au NCs and (ii) dopamine-conjugated Au NCs. $\lambda_{e x}=365 \mathrm{~nm}$. Concentration of Au NCs: $1.5 \mathrm{mg} / \mathrm{mL}$. (b) ECL spectra of (i) as-synthesized Au NCs and (ii) dopamine-conjugated Au NCs in the presence of $0.5 \mathrm{M}$ TEA as a co-reactant. Electrolyte: $0.1 \mathrm{M} \mathrm{KNO}_{3}$. Applied potential: $1.3 \mathrm{~V}$. Concentration of Au NCs: $0.75 \mathrm{mg} / \mathrm{mL}$.

\section{Conclusions}

In summary, here, we reported a post-synthesis modification of the shell of Au NCs with dopamine for tailoring PL and ECL of the NCs. The Au NCs were synthesized using GSH as a thiolate ligand and then subjected to the post-synthesis functionalization via amidic coupling of dopamine on the cluster shell. The post-synthesis functionalization of the as-synthesized Au NCs led to an increase in the PL with a maximum peak at $610 \mathrm{~nm}$ (i.e., orange PL), but the clusters' NIR ECL decreased. Combined with the findings from previous studies that the orange color PL and the NIR ECL of the Au NCs could be attributed to the $\mathrm{Au}(\mathrm{I})$-SG motifs on the cluster shell and the $\mathrm{Au}(0)$-SG motifs in the core of the NCs respectively $[30,31]$, the present study could rationalize both the increase in the clusters' orange PL and the decrease in their NIR ECL that we observed after we 
functionalized the cluster shell of the as-synthesized Au NCs with dopamine. The findings of the present study demonstrate the significance of the post-synthesis functionalization of the cluster shell as surface engineering of NCs to tailor the clusters' PL and ECL features. Furthermore, because the post-synthesis modification of the shell of Au NCs is a feasible and facile functionalization strategy of Au NCs with little dependency on any synthetic methods of the Au NCs, we envision that it can be applied to many different GSH-protected $\mathrm{Au}$ NCs for tailoring PL and ECL features of the clusters.

Supplementary Materials: The following are available online at https:/ / www.mdpi.com/2079-499 1/11/1/46/s1, Figure S1: UV-vis absorption spectrum of free dopamine, Figure S2: ${ }^{1} \mathrm{H}$ NMR spectra of (a) as-synthesized Au NCs and (b) dopamine-conjugated Au NCs. Figure S3: PL excitation spectra of (i) as-synthesized Au NCs and (ii) dopamine-conjugated Au NCs.

Author Contributions: Conceptualization, J.H.K. and J.K.; methodology, J.H.K. and J.K.; software, J.H.K.; validation, J.H.K. and J.K.; formal analysis, J.H.K.; investigation, J.H.K.; resources, J.K.; data curation, J.H.K. and J.K.; writing-original draft preparation, J.H.K. and J.K.; writing-review and editing, J.H.K. and J.K.; visualization, J.H.K.; supervision, J.K.; project administration, J.K.; funding acquisition, J.K. All authors have read and agreed to the published version of the manuscript.

Funding: This research was supported by the National Research Foundation of Korea funded by the Ministry of Science, ICT and Future Planning (NRF-2020R1A2C2013790 and NRF-2019M3E6A1065038).

Institutional Review Board Statement: Not applicable.

Informed Consent Statement: Not applicable.

Data Availability Statement: The data presented in this study are available on request from the corresponding author. The data are not publicly available due to institutional and funding regulations.

Conflicts of Interest: The authors declare no conflict of interest.

\section{References}

1. Jin, R.; Zeng, C.; Zhou, M.; Chen, Y. Atomically Precise Colloidal Metal Nanoclusters and Nanoparticles: Fundamentals and Opportunities. Chem. Rev. 2016, 116, 10346-10413. [CrossRef] [PubMed]

2. Cui, M.; Zhao, Y.; Song, Q. Synthesis, optical properties and applications of ultra-small luminescent gold nanoclusters. Trends Anal. Chem. 2014, 57, 73-82. [CrossRef]

3. Kaur, N.; Aditya, R.N.; Singh, A.; Kuo, T.-R. Biomedical Applications for Gold Nanoclusters: Recent Developments and Future Perspectives. Nanoscale Res. Lett. 2018, 13, 302. [CrossRef] [PubMed]

4. Chen, C.; Zhao, D.; Jiang, Y.; Ni, P.; Zhang, C.; Wang, B.; Yang, F.; Lu, Y.; Sun, J. Logically Regulating Peroxidase-Like Activity of Gold Nanoclusters for Sensing Phosphate-Containing Metabolites and Alkaline Phosphatase Activity. Anal. Chem. 2019, 91, 15017-15024. [CrossRef]

5. Latorre, A.; Latorre, A.; Castellanos, M.; Rodriguez Diaz, C.; Lazaro-Carrillo, A.; Aguado, T.; Lecea, M.; Romero-Pérez, S.; Calero, M.; Sanchez-Puelles, J.M.; et al. Multifunctional Albumin-Stabilized Gold Nanoclusters for the Reduction of Cancer Stem Cells. Cancers 2019, 11, 969. [CrossRef]

6. Lim, D.C.; Seo, B.Y.; Nho, S.; Kim, D.H.; Hong, E.M.; Lee, J.Y.; Park, S.-Y.; Lee, C.-L.; Kim, Y.D.; Cho, S. Emissive Nanoclusters Based on Subnanometer-Sized Au38 Cores for Boosting the Performance of Inverted Organic Photovoltaic Cells. Adv. Energy Mater. 2015, 5, 1500393. [CrossRef]

7. Zhang, X.; Chen, M.; Zhang, Y.; Hou, Y.; Wu, Y.; Yao, M.; Li, L.; Shi, L.; Liu, T.; Hu, B.; et al. Monoclonal-Antibody-Templated Gold Nanoclusters for HER2 Receptors Targeted Fluorescence Imaging. ACS Appl. Bio. Mater. 2020, 3, 7061-7066. [CrossRef]

8. Zhao, Q.; Huang, H.; Zhang, L.; Wang, L.; Zeng, Y.; Xia, X.; Liu, F.; Chen, Y. Strategy to Fabricate Naked-Eye Readout Ultrasensitive Plasmonic Nanosensor Based on Enzyme Mimetic Gold Nanoclusters. Anal. Chem. 2016, 88, 1412-1418. [CrossRef]

9. Wang, G.; Huang, T.; Murray, R.W.; Menard, L.; Nuzzo, R.G. Near-IR Luminescence of Monolayer-Protected Metal Clusters. J. Am. Chem. Soc. 2005, 127, 812-813. [CrossRef]

10. Miles, D.T.; Murray, R.W. Temperature-Dependent Quantized Double Layer Charging of Monolayer-Protected Gold Clusters. Anal. Chem. 2003, 75, 1251-1257. [CrossRef]

11. Fang, Y.-M.; Song, J.; Li, J.; Wang, Y.-W.; Yang, H.-H.; Sun, J.-J.; Chen, G.-N. Electrogenerated chemiluminescence from Au nanoclusters. Chem. Commun. 2011, 47, 2369-2371. [CrossRef] [PubMed]

12. Qian, H.; Zhu, M.; Wu, Z.; Jin, R. Quantum Sized Gold Nanoclusters with Atomic Precision. Acc. Chem. Res. 2012, 45, 1470-1479. [CrossRef] [PubMed]

13. Plascencia-Villa, G.; Demeler, B.; Whetten, R.L.; Griffith, W.P.; Alvarez, M.; Black, D.M.; José-Yacamán, M. Analytical Characterization of Size-Dependent Properties of Larger Aqueous Gold Nanoclusters. J. Phys. Chem. C 2016, 120, 8950-8958. [CrossRef] 
14. Li, G.; Hu, W.; Sun, Y.; Xu, J.; Cai, X.; Cheng, X.; Zhang, Y.; Tang, A.; Liu, X.; Chen, M.; et al. Reactivity and Lability Modulated by a Valence Electron Moving in and out of 25-Atom Gold Nanoclusters. Angew. Chem. Int. Ed. 2020, 59, 21135-21142. [CrossRef] [PubMed]

15. Negishi, Y.; Takasugi, Y.; Sato, S.; Yao, H.; Kimura, K.; Tsukuda, T. Magic-Numbered Aun Clusters Protected by Glutathione Monolayers $(n=18,21,25,28,32,39)$ : Isolation and Spectroscopic Characterization. J. Am. Chem. Soc. 2004, 126, 6518-6519. [CrossRef]

16. Wu, Z.; Jin, R. On the Ligand's Role in the Fluorescence of Gold Nanoclusters. Nano Lett. 2010, 10, 2568-2573. [CrossRef]

17. Schaaff, T.G.; Knight, G.; Shafigullin, M.N.; Borkman, R.F.; Whetten, R.L. Isolation and Selected Properties of a 10.4 kDa Gold:Glutathione Cluster Compound. J. Phys. Chem. B 1998, 102, 10643-10646. [CrossRef]

18. Luo, Z.; Yuan, X.; Yu, Y.; Zhang, Q.; Leong, D.T.; Lee, J.Y.; Xie, J. From Aggregation-Induced Emission of Au(I)-Thiolate Complexes to Ultrabright $\mathrm{Au}(0) @ \mathrm{Au}(\mathrm{I})-$ Thiolate Core-Shell Nanoclusters. J. Am. Chem. Soc. 2012, 134, 16662-16670. [CrossRef]

19. Negishi, Y.; Nobusada, K.; Tsukuda, T. Glutathione-Protected Gold Clusters Revisited: Bridging the Gap between Gold(I)-Thiolate Complexes and Thiolate-Protected Gold Nanocrystals. J. Am. Chem. Soc. 2005, 127, 5261-5270. [CrossRef]

20. Yu, Y.; Luo, Z.; Chevrier, D.M.; Leong, D.T.; Zhang, P.; Jiang, D.-E.; Xie, J. Identification of a Highly Luminescent Au $22($ SG) 18 Nanocluster. J. Am. Chem. Soc. 2014, 136, 1246-1249. [CrossRef]

21. Pyo, K.; Thanthirige, V.D.; Kwak, K.; Pandurangan, P.; Ramakrishna, G.; Lee, D. Ultrabright Luminescence from Gold Nanoclusters: Rigidifying the Au(I)-Thiolate Shell. J. Am. Chem. Soc. 2015, 137, 8244-8250. [CrossRef] [PubMed]

22. Sun, Y.; Wu, J.; Wang, C.; Zhao, Y.; Lin, Q. Tunable near-infrared fluorescent gold nanoclusters: Temperature sensor and targeted bioimaging. New J. Chem. 2017, 41, 5412-5419. [CrossRef]

23. Peng, H.; Huang, Z.; Sheng, Y.; Zhang, X.; Deng, H.; Chen, W.; Liu, J. Pre-oxidation of Gold Nanoclusters Results in a $66 \%$ Anodic Electrochemiluminescence Yield and Drives Mechanistic Insights. Angew. Chem. Int. Ed. 2019, 58, 11691-11694. [CrossRef] [PubMed]

24. Cantelli, A.; Guidetti, G.; Manzi, J.; Caponetti, V.; Montalti, M. Towards Ultra-Bright Gold Nanoclusters. Eur. J. Inorg. Chem. 2017, 2017, 5068-5084. [CrossRef]

25. Genji Srinivasulu, Y.; Yao, Q.; Goswami, N.; Xie, J. Interfacial engineering of gold nanoclusters for biomedical applications. Mater. Horiz. 2020, 7, 2596-2618. [CrossRef]

26. Pyo, K.; Thanthirige, V.D.; Yoon, S.Y.; Ramakrishna, G.; Lee, D. Enhanced luminescence of $\mathrm{Au}_{22}(\mathrm{SG})_{18}$ nanoclusters via rational surface engineering. Nanoscale 2016, 8, 20008-20016. [CrossRef]

27. Tang, Z.; Ahuja, T.; Wang, S.; Wang, G. Near infrared luminescence of gold nanoclusters affected by the bonding of 1,4-dithiolate durene and monothiolate phenylethanethiolate. Nanoscale 2012, 4, 4119-4124. [CrossRef]

28. Montalti, M.; Zaccheroni, N.; Prodi, L.; O’Reilly, N.; James, S.L. Enhanced Sensitized NIR Luminescence from Gold Nanoparticles via Energy Transfer from Surface-Bound Fluorophores. J. Am. Chem. Soc. 2007, 129, 2418-2419. [CrossRef]

29. Yahia-Ammar, A.; Sierra, D.; Mérola, F.; Hildebrandt, N.; Le Guével, X. Self-Assembled Gold Nanoclusters for Bright Fluorescence Imaging and Enhanced Drug Delivery. ACS Nano 2016, 10, 2591-2599. [CrossRef]

30. Kim, J.M.; Jeong, S.; Song, J.K.; Kim, J. Near-infrared electrochemiluminescence from orange fluorescent Au nanoclusters in water. Chem. Commun. 2018, 54, 2838-2841. [CrossRef]

31. Kang, Y.; Kim, J. Electrochemiluminescence of Glutathione-Stabilized Au Nanoclusters Fractionated by Gel Electrophoresis in Water. ChemElectroChem 2020, 7, 1092-1096. [CrossRef]

32. Zhang, Q.; Xie, J.; Yu, Y.; Lee, J.Y. Monodispersity control in the synthesis of monometallic and bimetallic quasi-spherical gold and silver nanoparticles. Nanoscale 2010, 2, 1962-1975. [CrossRef] [PubMed]

33. Basu, S.; Panigrahi, S.; Praharaj, S.; Kumar Ghosh, S.; Pande, S.; Jana, S.; Pal, T. Dipole-dipole plasmon interactions in self-assembly of gold organosol induced by glutathione. New J. Chem. 2006, 30, 1333-1339. [CrossRef]

34. Wu, Z.; Gayathri, C.; Gil, R.R.; Jin, R. Probing the Structure and Charge State of Glutathione-Capped Au ${ }_{25}(\mathrm{SG})_{18}$ Clusters by NMR and Mass Spectrometry. J. Am. Chem. Soc. 2009, 131, 6535-6542. [CrossRef] [PubMed]

35. Wu, Z.; Jin, R. Stability of the Two Au-S Binding Modes in $\mathrm{Au}_{25}(\mathrm{SG})_{18}$ Nanoclusters Probed by NMR and Optical Spectroscopy. ACS Nano 2009, 3, 2036-2042. [CrossRef] [PubMed]

36. Yu, Y.; Chen, X.; Yao, Q.; Yu, Y.; Yan, N.; Xie, J. Scalable and Precise Synthesis of Thiolated Au10-12, Au15, Au18, and Au25 Nanoclusters via pH Controlled CO Reduction. Chem. Mater. 2013, 25, 946-952. [CrossRef]

37. Liebscher, J.; Mrówczyński, R.; Scheidt, H.A.; Filip, C.; Hădade, N.D.; Turcu, R.; Bende, A.; Beck, S. Structure of Polydopamine: A Never-Ending Story? Langmuir 2013, 29, 10539-10548. [CrossRef]

38. Lyu, Q.; Song, H.; Yakovlev, N.L.; Tan, W.S.; Chai, C.L.L. In situ insights into the nanoscale deposition of 5,6-dihydroxyindolebased coatings and the implications on the underwater adhesion mechanism of polydopamine coatings. RSC Adv. 2018, 8 , 27695-27702. [CrossRef]

39. Goswami, N.; Lin, F.; Liu, Y.; Leong, D.T.; Xie, J. Highly Luminescent Thiolated Gold Nanoclusters Impregnated in Nanogel. Chem. Mater. 2016, 28, 4009-4016. [CrossRef]

40. Gao, F.; Qu, H.; Duan, Y.; Wang, J.; Song, X.; Ji, T.; Cao, L.; Nie, G.; Sun, S. Dopamine coating as a general and facile route to biofunctionalization of superparamagnetic $\mathrm{Fe}_{3} \mathrm{O}_{4}$ nanoparticles for magnetic separation of proteins. RSC Adv. 2014, 4, 6657-6663. [CrossRef] 
41. Bachman, R.E.; Bodolosky-Bettis, S.A.; Glennon, S.C.; Sirchio, S.A. Formation of a Novel Luminescent Form of Gold(I) Phenylthiolate via Self-Assembly and Decomposition of Isonitrilegold(I) Phenylthiolate Complexes. J. Am. Chem. Soc. 2000, 122, 7146-7147. [CrossRef]

42. Pyo, K.; Ly, N.H.; Han, S.M.; Hatshan, M.b.; Abuhagr, A.; Wiederrecht, G.; Joo, S.-W.; Ramakrishna, G.; Lee, D. Unique Energy Transfer in Fluorescein-Conjugated Au22 Nanoclusters Leading to 160-Fold pH-Contrasting Photoluminescence. J. Phys. Chem. Lett. 2018, 9, 5303-5310. [CrossRef] [PubMed]

43. Huang, Z.; Wang, M.; Guo, Z.; Wang, H.; Dong, H.; Yang, W. Aggregation-Enhanced Emission of Gold Nanoclusters Induced by Serum Albumin and Its Application to Protein Detection and Fabrication of Molecular Logic Gates. ACS Omega 2018, 3, 12763-12769. [CrossRef] [PubMed]

44. You, J.G.; Tseng, W.L. Peptide-induced aggregation of glutathione-capped gold nanoclusters: A new strategy for designing aggregation-induced enhanced emission probes. Anal. Chim. Acta 2019, 1078, 101-111. [CrossRef] [PubMed]

45. Xue, J.-H.; Xiao, K.-P.; Wang, Y.-S.; Liu, L.; Li, J.-Q.; Li, M.; Qu, Y.-N.; Xiao, X.-L. Aggregation-induced photoluminescence enhancement of protamine-templated gold nanoclusters for 1-hydroxypyrene detection using 9-hydroxyphenanthrene as a sensitizer. Colloids Surf. B 2020, 189, 110873. [CrossRef] [PubMed] 